\title{
DINÂMICA DOS ATRIBUTOS QUÍMICOS DO SOLO AO LONGO DA SAFRA 2008/2009, PARA SEIS CULTIVARES DE CANA-DE-AÇÚCAR
}

\author{
SILVA NETO, Hélio Francisco ${ }^{1}$ \\ SILVA, Joana Diniz Rosa ${ }^{2}$ \\ TASSO JÚNIOR, Luiz Carlos ${ }^{3}$ \\ MARQUES, Diogo ${ }^{4}$ \\ MARQUES, Marcos Omir ${ }^{5}$
}

\begin{abstract}
RESUMO: A análise química do solo permite avaliar parâmetros responsáveis pelo melhor desenvolvimento da cana-de-açúcar, possibilitando a adequação da cultura às condições do solo em que ela é inserida. O solo cultivado com os genótipos de cana-de-açúcar apresenta dinâmica diferente ao longo do tempo de cultivo. Sendo assim, o objetivo deste trabalho foi comparar a composição química do solo, na camada 0-20 $\mathrm{cm}$ de profundidade, sob seis cultivares de cana-de-açúcar, ao longo da safra 2008/2009. O experimento foi desenvolvido na FCAV/UNESP, Jaboticabal. O delineamento experimental utilizado foi em blocos casualizados em esquema fatorial $6 \times 3$ com 3 repetições. Os tratamentos principais formam os seis cultivares com ciclo de maturação no meio de safra: CTC 15, IAC91-1099, IACSP94-4004, IACSP95-5000, RB855536 e SP81-3250. Os tratamentos secundários foram as três análises realizadas ao longo da safra: 12, 15 e 17 meses após o plantio. A amostragem de solo ocorreu de forma aleatória nas entrelinhas da cana. As amostras foram enviadas ao Laboratório para análise química de solo. Pode-se concluir que os teores de matéria orgânica, pH e CTC no solo tendem a diminuir ao longo das épocas analisadas. Comportamento inverso foi observado para acidez potencial. Os cultivares IACSP94-4004 e RB855536 obtiveram os resultados mais satisfatórios apresentando elevados teores de matéria orgânica, $\mathrm{pH}$ e CTC e baixa acidez potencial. Resultados contrários foram obtidos pelos cultivares CTC 15 e SP81-3250, indicando a pior qualidade do solo cultivado com estes exemplares, sendo necessário uma maior atenção para os próximos anos de cultivo da cana soca.
\end{abstract}

Palavras-chave: Adubação. Saccharum spp. Variedades.

\section{SOIL CHEMICAL ATRIBUTES DYNAMIC ALONG 2008/2009 HARVEST, FOR SIX SUGARCANE VARIETIES}

SUMMARY: The chemical soil analysis allows evaluation of parameters responsible for the better sugarcane development, enabling the crop adequacy to the soil conditions in which it is inserted. The cultivated soil with the sugarcane genotypes shows a dynamic different along the crop time. Thus, the objective of this work was to compare the soil chemical composition, at the $0-20 \mathrm{~cm}$ depth layer, under six sugarcane varieties, along the 2008/2009 harvest. The experiment was developed at FCAV/UNESP, Jaboticabal. The experimental design used was in randomized blocks in factorial scheme $6 \times 3$ with 3 replications. The main treatments were the six varieties with ripeness cycle in the middle of the harvest: CTC 15, IAC91-1099, IACSP94-4004, IACSP95-5000,

\footnotetext{
${ }^{1}$ Bolsista CNPq - Doutorando em Produção Vegetal pela Faculdade de Ciências Agrárias e Veterinárias FCAV UNESP/Jaboticabal. E-mail: heliofsn @ hotmail.com

${ }^{2}$ Bolsista CAPES - Mestranda em Produção Vegetal pela Faculdade de Ciências Agrárias e Veterinárias FCAV UNESP/Jaboticabal. E-mail: jodiniz2@yahoo.com.br

${ }^{3}$ Bolsista CAPES - Pós-doutorando em Produção Vegetal pela Faculdade de Ciências Agrárias e Veterinárias FCAV - UNESP/Jaboticabal. E-mail: lctasso@ yahoo.com.br

${ }^{4}$ Departamento de Tecnologia.Laboratório do Açúcar e Etanol. UNESP/FCAV, campus Jaboticabal

${ }^{5}$ Professor Adjunto do Departamento de Tecnologia da Faculdade de Ciências Agrárias e Veterinárias FCAV UNESP/Jaboticabal. E-mail: omir@ fcav.unesp.br
} 
RB855536 and SP81-3250. The secondary treatments were the three analysis performed along the harvest: 12 , 15 and 17 months after planting. The soil sampling happened in random form in the sugarcane interlines. The samples were sent to the Laboratory of the Soil and Fertilizers Department at FCAV for soil chemical analysis. It can be concluded that organic matter, $\mathrm{pH}$ and CEC levels in the soil tend to decrease along the analyzed epochs. Inverse behavior was observed for potential acidity. The varieties IACSP94-4004 and RB855536 had the most satisfactory results showing higher organic matter, $\mathrm{pH}$ and CEC levels and low potential acidity. Opposite results were obtained with CTC 15 and SP81-3250 varieties, showing the worst soil quality when cultivated with these copies, becoming necessary more attention for the next years of second ripening cane harvest.

Keywords: Fertilization. Saccharum spp. Varieties

\section{INTRODUÇÃO}

O conhecimento da análise química de solo, associado ao comportamento dos diferentes de cana-de-açúcar proporcionam a obtenção de melhores práticas de manejo e, consequentemente, maior eficiência na produção e gestão da cultura da cana-de-açúcar (TASSO JÚNIOR et al., 2010). Sendo assim, a partir desse conhecimento é possível se determinar quais medidas a serem tomadas para que a correção e adubação do solo sejam adequadas tanto para o desenvolvimento da cultura como para o âmbito econômico (CRESTE et al., 2008).

Os parâmetros do solo como matéria orgânica, $\mathrm{pH}$, acidez potencial e capacidade de troca catiônica devem ser quantificados, analisados e corrigidos, quando necessários, pois interferem na produtividade de cana (LANDELL et al., 2003). A matéria orgânica (M.O.) exerce influencia na disponibilidade de nutrientes do solo, afetando a ciclagem e adsorção destes elementos, podendo evitar ou diminuir a lixiviação dos mesmos. O aumento do teor de matéria orgânica pode indicar manejo adequado do solo, enquanto baixos teores indicam déficit na produção de resíduos vegetais, baixa fertilidade, excesso de revolvimento do solo, alta taxa de erosão, entre outros fatores (MIELNICZUK, 2008).

Marinho; Alburquerque (1984) estabeleceram quatro classes de acidez a serem consideradas para a cultura da cana-de-açúcar, sendo que $\mathrm{pH}$ abaixo de 4,6 é considerada acidez muito alta; pH entre 4,6 a 5,2 é considerada acidez alta; $\mathrm{pH}$ entre 5,3 a 5,6, acidez média; e pH acima de 5,6 a acidez é baixa.

Em condições de alta concentração de acidez potencial $(\mathrm{H}+\mathrm{Al})$ nos solos o crescimento de raízes fica restrito, sendo que estas se tornam grossas e curtas, devido à redução do crescimento de células e da divisão celular (MALAVOLTA et al., 1997). A capacidade de troca catiônica (CTC) de um solo depende de sua textura, do tipo de argila do solo e do teor de matéria orgânica, sendo que para o cultivo da cana-de-açúcar, a CTC deve estar acima de 55 mmolc $\mathrm{dm}^{-3}$ (PRADO et al., 1993). 
Além disso, os atributos químicos do solo, cultivado com diversos genótipos de canade-açúcar, apresentam variações ao longo da safra, devido à dinâmica do solo (TASSO JÚNIOR et al, 2010). Dessa forma, este trabalho teve como objetivo comparar a composição química do solo (M.O., pH, H+Al e CTC) na camada 0-20 cm de profundidade, sob seis cultivares de cana-de-açúcar, ao longo da safra 2008/2009.

\section{MATERIAL E MÉTODOS}

O experimento foi realizado na Fazenda de Ensino, Pesquisa e Produção (FEPP) da FCAV/UNESP, localizada no Município de Jaboticabal, Estado de São Paulo, Brasil. A altitude média é de 575 metros do nível do mar, com relevo caracterizado como suave ondulado. Sua localização geográfica é definida como: latitude $21^{\circ} 15^{\prime}, 22^{\prime}$ 'S e longitude $48^{\circ} 18^{\prime} 58^{\prime}$, WG. A região de clima tropical com inverno seco, e classificado, de acordo como o Sistema Internacional de Classificação de Köppen, como Aw. A pluviometria média anual é de $1425 \mathrm{~mm}$, com concentração de chuvas no verão.

O delineamento experimental utilizado foi em blocos casualizados em esquema fatorial $6 \times 3$ com 3 repetições. Os tratamentos principais foram os seis cultivares de cana-de-açúcar estudados (CTC 15, IAC91-1099, IACSP944004, IACSP95-5000, RB855536 e SP81-3250) e o secundário as 3 épocas analisadas ao longo da safra (12, 15 e 17 meses após o plantio).

O solo é classificado como um Latossolo-Vermelho Escuro, eutrófico, A moderado, textura argilosa e relevo suave ondulado - EUTRUSTOX (EMBRAPA, 1999). O preparo do solo foi efetuado no sistema convencional, com uma aração com arado de disco e duas gradagens, sendo uma grade aradora de $270 \mathrm{~mm}$ e uma grade niveladora de $195 \mathrm{~mm}$.

Para caracterização inicial do solo, foi realizada a coleta de 20 subamostras, procurando-se abranger toda a área na profundidade 0-20 e 20-40 centímetros. Após coletada, esta amostra foi submetida à secagem à sombra, peneirada e enviada para realização de análises químicas e físicas no

Departamento de Solos e Adubos da FCAV/UNESP (Tabelas 1 e 2). Segundo os critérios de Raij et al., (1996a) se trata de um solo argiloso com acidez média, com alto valor de potássio, cálcio, magnésio e fósforo, e média 
saturação das bases. De acordo com as recomendações de Raij et al., (1996b) não foi necessário realizar a correção do solo.

O plantio dos cultivares de cana foi realizado em março de 2007 . Na distribuição das mudas no sulco de plantio adotou-se o sistema de colmos cruzados, procurando atingir média de 18 gemas por metro linear, sendo que nesta ocasião, foi utilizado, como adubação de plantio $500 \mathrm{~kg} \mathrm{ha}^{-1} \mathrm{da}$ fórmula 05-25-25, seguindo critérios da perspectiva de produtividade agrícola e análise do solo, de acordo com Boletim 100 - IAC (SPIRONELLO et al., 1996).

Tabela 1. Propriedades químicas do solo da área experimental. Jaboticabal, SP, ano agrícola 2007/2008.

\begin{tabular}{|c|c|c|c|c|c|c|c|c|c|}
\hline tras & $\begin{array}{l}\mathrm{pH} \\
\mathrm{CaCl}_{2}\end{array}$ & $\begin{array}{l}\mathrm{P} \text { (resina) } \\
\mathrm{mg} \mathrm{dm}^{-3}\end{array}$ & $\begin{array}{l}\text { M.O. } \\
\text { g dm}^{-3}\end{array}$ & $\mathrm{~K}$ & $\mathrm{Ca}$ & $\begin{array}{l}\mathrm{Mg} \mathrm{H}+\mathrm{Al} \\
-\quad \mathrm{mmolc} \mathrm{dm}^{-3}\end{array}$ & SB & CTC & $\begin{array}{l}\mathrm{V} \% \\
\%\end{array}$ \\
\hline 0 & 5,3 & 22 & 19 & 3,8 & 37 & 31 & 56 & 87,8 & 65 \\
\hline $20-40$ & 5,3 & 18 & 15 & 3,5 & 28 & 12 & 43,5 & 68,5 & 64 \\
\hline
\end{tabular}

Fonte: Departamento de Solos e Adubos - FCAV/UNESP

Tabela 2. Propriedades granulométricas do solo da área experimental. Jaboticabal, SP, ano agrícola 2007/2008.

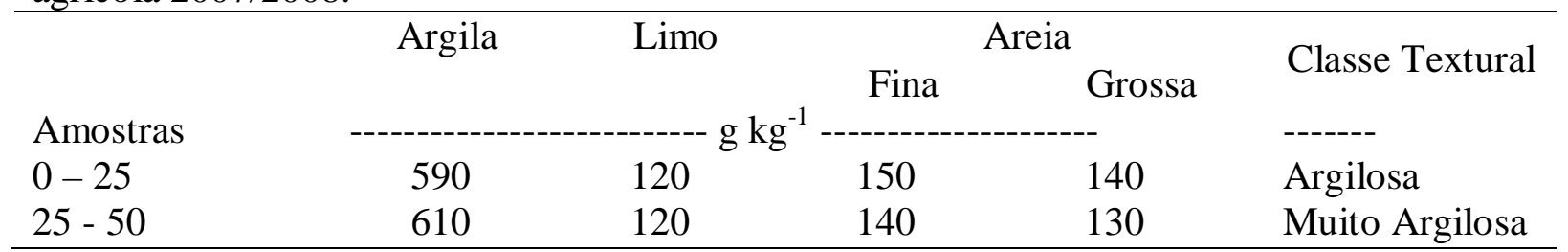

Fonte: Departamento de Solos e Adubos - FCAV/UNESP

No campo o experimento era constituído por 3 blocos em cada blocos os cultivares eram representados por parcelas dispostas ao acaso. Cada parcela era composta por cinco linhas de cana, com 12 metros de comprimento, espaçadas de 1,5 metros entre elas, totalizando $90 \mathrm{~m}^{2}$. Para a retirada de amostras de solo foram descartadas a primeira e a quinta linha, e 1 metros das extremidades das três linhas centrais, totalizado $45 \mathrm{~m}^{2}$.

Foram coletadas 20 sub-amostras de solo na profundidade $0-20 \mathrm{~cm}$ na entrelinha da cana, em cada parcela, sendo o local de coleta, escolhido aleatoriamente no momento da amostragem. Após coletadas, as sub-amostras foram homogeneizadas e deram origem à amostra composta, a qual foi etiquetada e encaminhada para o Laboratório de análise química do solo do Departamento de Solos e Adubos da FCAV/UNESP. 
No laboratório foram determinados os valores de CTC, segundo método preconizado por Raij; Quaggio (2001b), matéria orgânica (RAIJ; QUAGGIO, 2001a) e os valores de pH e H+Al (RAIJ; QUAGGIO, 2001c). Os resultados obtidos foram submetidos à análise de variância pelo teste $F$, e quando significativo as médias foram comparadas pelo teste de Tukey ao nível de 5\% de probabilidade, conforme procedimento proposto por Pimentel Gomes; Garcia (2002).

\section{RESULTADOS E DISCUSSÃO}

Na Tabela 3 estão apresentados os valores de matéria orgânica (M.O.), pH, acidez potencial $(\mathrm{H}+\mathrm{Al})$ e capacidade de troca catiônica (CTC) obtidos para os cultivares estudados. Ao analisar os teores de matéria orgânica, os cultivares RB855536 e IACSP94-4004 obtiveram os maiores valores, proporcionando assim um solo de maior fertilidade, com menor ocorrência de erosão e lixiviação de macro e micronutrientes (MIELNICZUK, 2008).

Embora constatado o aumento nos teores de matéria orgânica entre as épocas 1 e 2, (12 e 15 meses após o plantio, respectivamente) foi observado ao final do ciclo diminuição dos valores de M.O. Esta tendência de redução ao longo da safra pode ser observada pela interação obtida entre cultivares e épocas de análise, principalmente pelos cultivares SP813250 e RB855536 (Figura 1).

Os menores valores de $\mathrm{pH}$ foram obtidos pelo solo dos cultivares CTC 15 e SP813250, porém estes menores valores de $\mathrm{pH}$ são considerados médios, pela classificação de Marinho; Albuquerque (1984). Os demais cultivares apresentaram solos de acidez média.

Os cultivares CTC 15 e SP81-3250 obtiveram também as maiores concentrações de $\mathrm{H}+\mathrm{Al}$, evidenciando a relação que há entre a acidez potencial e o $\mathrm{pH}$ do solo, seja, quanto menor o pH maior será a acidez potencial. De acordo com Malavolta et al. (1997), estes maiores valores de acidez potencial encontrados no solo cultivado com estes exemplares de cana poderiam ocasionar problemas no desenvolvimento de suas raízes, que podem ficar grossas e curtas, devido a diminuição da divisão e crescimento celular. 
Tabela 3. Valores médios ${ }^{1}$ dos teores de matéria orgânica (M.O.), pH, capacidade de troca catiônica (CTC) e acidez potencial $(\mathrm{H}+\mathrm{Al})$ obtidos pelos solos $(0-20 \mathrm{~cm})$ dos cultivares de cana-de-açúcar, ao longo da safra 2008/2009.

\begin{tabular}{|c|c|c|c|c|}
\hline Tratamentos & $\begin{array}{l}\text { M.O. } \\
\mathrm{g} \mathrm{dm}^{-3}\end{array}$ & \multirow[t]{2}{*}{$\begin{array}{c}\mathbf{p H} \\
\mathrm{CaCl}_{2} \\
\end{array}$} & \multicolumn{2}{|c|}{$\begin{array}{c}\mathbf{H}+\mathbf{A l} \mathbf{C T C} \\
---- \text { mmolc }^{-3}{ }^{-3}---- \\
\end{array}$} \\
\hline \multicolumn{4}{|l|}{ Cultivares (C) } & \\
\hline CTC 15 & $20,00 \mathrm{c}$ & $5,34 \mathrm{~d}$ & 29,66 a & $73,17 \mathrm{e}$ \\
\hline IAC91-1099 & $21,33 \mathrm{~b}$ & $5,66 \mathrm{c}$ & $27,55 \mathrm{~b}$ & $82,85 \mathrm{~d}$ \\
\hline IACSP94-4004 & $23,88 \mathrm{a}$ & $5,92 \mathrm{a}$ & $22,22 \mathrm{c}$ & $103,20^{\mathrm{a}}$ \\
\hline IACSP95-5000 & $21,00 \mathrm{bc}$ & $5,60 \mathrm{c}$ & $27,55 \mathrm{~b}$ & $87,04 \mathrm{c}$ \\
\hline RB855536 & $24,77 \mathrm{a}$ & $5,73 \mathrm{~b}$ & $23,11 \mathrm{c}$ & $94,911 \mathrm{~b}$ \\
\hline SP81-3250 & $20,22 \mathrm{bc}$ & $5,36 \mathrm{~d}$ & $30,00 \mathrm{a}$ & $87,85 \mathrm{c}$ \\
\hline $\operatorname{DMS}(5 \%)$ & 1,31 & 0,12 & 1,59 & 2,22 \\
\hline \multicolumn{5}{|l|}{ Época $(E)$} \\
\hline 1 & $22,72 b$ & $5,68 \mathrm{a}$ & $24,77 \mathrm{~b}$ & $94,96 \mathrm{a}$ \\
\hline 2 & $23,611 \mathrm{a}$ & $5,69 \mathrm{a}$ & $25,27 \mathrm{~b}$ & $88,65 b$ \\
\hline 3 & $19,27 \mathrm{c}$ & $5,38 b$ & $30,00 \mathrm{a}$ & $80,90 \mathrm{c}$ \\
\hline DMS (5\%) & 0,75 & 0,07 & 0,91 & 1,27 \\
\hline \multicolumn{5}{|c|}{ Estatística - Teste F } \\
\hline Cultivares (C) & $42,10 * *$ & $54,34 * *$ & $77,24 * *$ & $385,95 * *$ \\
\hline Época $(E)$ & $111,44 * *$ & $71,26 * *$ & $118,59 * *$ & $364,12 * *$ \\
\hline Interação (CxE) & $9,54 * *$ & $6,20 * *$ & $16,18 * *$ & $76,91 * *$ \\
\hline Blocos & $4,96^{*}$ & $2,98^{\mathrm{ns}}$ & $2,84^{\mathrm{ns}}$ & $0,84^{\mathrm{ns}}$ \\
\hline $\mathrm{CV} \%$ & 4,20 & 1,60 & 4,20 & 1,77 \\
\hline
\end{tabular}

Médias seguidas da mesma letra na coluna, em cada atributo, não diferem entre si pelo teste de Tukey. ${ }^{\text {ns }},{ }^{*} \mathrm{e}^{* *}$ Não significativo e significativo ao nível de 5 e $1 \%$ de probabilidade, pelo teste F, respectivamente. DMS diferença mínima significativa, $\mathrm{CV}$ - coeficiente de variação.

Ao longo do tempo de cultivo foi observado aumento da acidez do solo, caracterizada pela diminuição do $\mathrm{pH}$ e aumento da acidez potencial. O solo sob os cultivares CTC 15 e SP81-3250 apresentaram dinâmica diferente em relação aos demais. Resultados semelhantes foram obtidos por Tasso Júnior et al., (2010) que também constataram comportamento diferentes entre o solo dos cultivares estudados.

O solo dos cultivares apresentaram diminuição dos valores de CTC ao longo do tempo analisado. No entanto, o cultivar IACSP94-4004 obteve comportamento diferente, sendo observado acréscimo entre as épocas 2 e 3 (15 e 17 meses após o plantio, respectivamente). Tal resultado discorda de Moreti (2002), uma vez que eles não acompanham os resultados de matéria orgânica e pH ao longo da safra. Porém, a relação entre matéria orgânica, pH e CTC pode ser observada em todos os outros cultivares, onde o decréscimo dos valores de $\mathrm{pH}$ e matéria orgânica resultam num decréscimo de CTC. Os valores de CTC encontrados estão acima de 55 mmolc $\mathrm{dm}^{-3}$, sendo suficientes para a cultura da cana-de-açúcar, para todos os cultivares e épocas estudados (PRADO et al., 1993). 

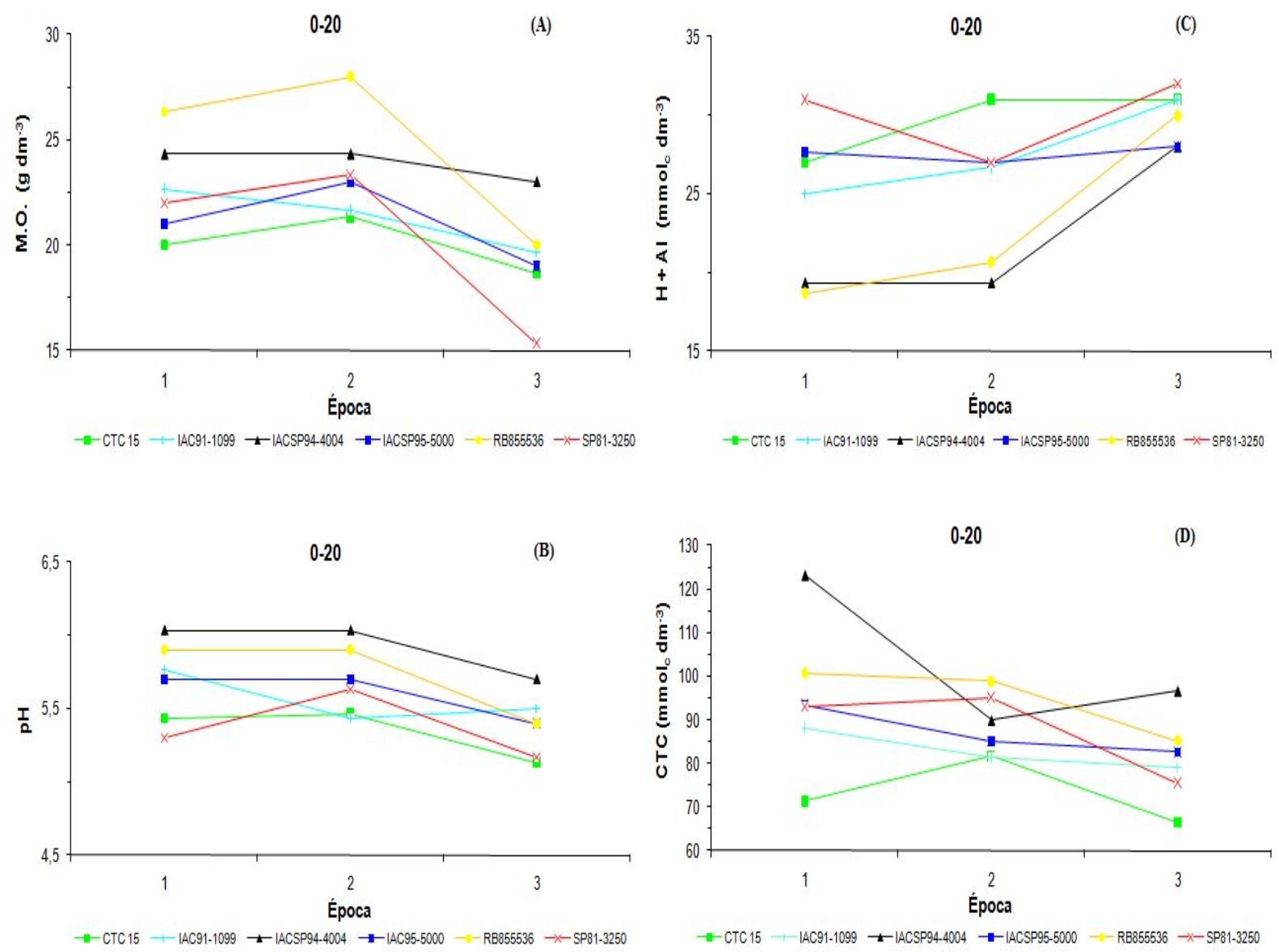

Figura 1. Desdobramento dos cultivares e épocas sobre o teor de matéria orgânica (A), valor de $\mathrm{pH}(\mathrm{B})$, acidez potencial (C) e capacidade de troca catiônica (D) na profundidade de solo analisada $(0-20 \mathrm{~cm})$.

\section{CONCLUSÃO}

A partir deste trabalho pode-se concluir que os teores de matéria orgânica, pH e CTC no solo tendem a diminuir ao longo das épocas analisadas. Comportamento inverso foi observado para acidez potencial.

Os cultivares IACSP94-4004 e RB855536 obtiveram os resultados mais satisfatórios apresentando elevados teores de matéria orgânica, pH e CTC e baixa acidez potencial. Resultados contrários foram obtidos pelos cultivares CTC 15 e SP81-3250, indicando a pior qualidade do solo cultivado com estes exemplares, sendo necessário uma maior atenção para os próximos anos de cultivo da cana soca. 


\section{REFERÊNCIAS}

CRESTE, S.; JUNIOR, V. E. R.; PINTO, L. R.; ALBINO, J. C.; FIGUEIRA, A. V. O. A biotecnologia como ferramenta para o melhoramento genético. In: DINARDO MIRANDA, L. L.; VASCONCELOS, A. C. M. de; LANDELL, M. G. A. (Eds). Cana-de-açúcar. Campinas: Instituto Agronômico, 2008. part. 3, cap. 6, p. 170.

EMBRAPA - Empresa Brasileira de Pesquisa Agropecuária. Sistema brasileiro de classificação de solos. Brasília: EMBRAPA Solos, Centro Nacional de Pesquisa de Solos, 1999. 412p.

LANDELL, M.G.A.; PRADO, H.; VASCONCELOS, A.C.M.; PERECIN, D.; ROSSETTO, R.; BIDÓIA, M.A.P.; SILVA, M.A.; XAVIER, M.A. Oxisol subsurface chemical attributes related to sugarcane productivity. Scientia Agricola, Piracicaba, v. 60, p. 741-745, 2003.

MALAVOLTA, E.; VITTI, G.C.; OLIVEIRA, S. A. Avaliação do estado nutricional das plantas. Princípios e aplicações. 2. ed. Piracicaba, Potafos, 1997, 319p.

MARINHO, M.L.; ALBUQUERQUE, G.A.C. Efeito do calcário dolomítico sobre a produção de cana-de-açúcar em Alagoas, medidos pelo pH do solo. Cadernos Planalsucar, Piracicaba, v. 1, p. 19-23. 1984.

MIELNICZUK, J. Matéria Orgânica e Sustentabilidade de Sistemas Agrícolas. In: santos, G.A.; SILVA, L.S.; CANELLAS, L.P.; CAMARGO, F. A. O. Fundamentos da matéria orgânica do solo: ecossistemas tropicais e subtropicais. 2. ed. Porto Alegre: Metrópole, 2008. p. 1-18.

MORETI, D. Propriedades físicas e químicas de um latossolo vermelho cultivado com feijão e algodão, sob diferentes sistemas de preparo, adubações e plantas de cobertura 2002. 85p. Dissertação (Mestrado em Agronomia) - Faculdade de Ciências Agrárias e Veterinárias, Universidade Estadual Paulista, Jaboticabal.

PIMENTEL-GOMES, F.; GARCIA, C.H.. Estatística aplicada a experimentos agronômicos e florestais: exposição com exemplos e orientações pra uso de aplicativos. Piracicaba, FEALQ, 2002, 309 p.

PRADO, H. do; PÁDUA JÚNIOR, A. L.; GARCIA, J. C.; MORAES, J. F. L. de; CARVALHO, J. P. de; DONZELI, P. L. Solos e ambiente de produção. In DINARDOPENATTI, C. P.: FORTI, J. A. Projeto: Calcário e Gesso em cana-de-açúcar. COPERSUCAR, 1993, 79p.

RAIJ, B. van QUAGGIO, J. A.; CANTARELLA, H.; ABREU, C. A. Interpretação de resultados de análise de solo. In: RAIJ, B van; CANTARELLA, H.; QUAGGIO, J. A.; FURLANI, A. M. C. Recomendações de adubação e calagem para o Estado de São Paulo. 2 ed. Campinas: Instituto Agronômico, 1996a. p. 8-13 (Boletim Técnico 100).

RAIJ, B., van.; CANTARELlA, H.; QUAGGIO, J., A., FURLANI A., M., C., Recomendações de adubação e calagem para o estado de São Paulo In: RAIJ, B. van; CANTARELLA, H.; QUAGGIO J.A.; FURLANI A.M.C. Recomendações de adubação e calagem para o Estado de São Paulo. 2 ed. Campinas: Instituto Agronômico, 1996b. p. 3-6 (Boletim Técnico 100). 
RAIJ, B. van; QUAGGIO, J. A. Determinação da matéria orgânica. In: RAIJ B. van; ANDRADE J. C.; CANTARELLA H.; QUAGGIO, J. A. Análise química para avaliação da fertilidade de solos tropicais, Campinas: Instituto Agronômico, 2001a. p. 173-180.

RAIJ, B. van; QUAGGIO, J. A. Determinação de fósforo, cálcio, magnésio e potássio extraídos com resina trocadora de íons. In: RAIJ B. van; ANDRADE J. C.; CANTARELLA H.; QUAGGIO, J. A. Análise química para avaliação da fertilidade de solos tropicais, Campinas: Instituto Agronômico, 2001b. p.189-199.

RAIJ, B. van; QUAGGIO, J. A. Determinação do pH em cloreto de cálcio e da acidez total. In: RAIJ B. van; ANDRADE J. C.; CANTARELLA H.; QUAGGIO, J. A. Análise química para avaliação da fertilidade de solos tropicais, Campinas: Instituto Agronômico, 2001c. p. 181-188.

SPIRONELLO, A.; RAIJ, B. van.; PENATTI, C. P.; CANTARELLA, H.; MORELLI, J. L.; ORLANDO FILHO, J.; LANDELL, M. G. A.; ROSSETO, R. Cana-de-açúcar. In: RAIJ, B. van; CANTARELLA , H.; QUAGGIO J.A.; FURLANI A.M.C. Recomendações de adubação e calagem para o Estado de São Paulo. 2 ed. Campinas: Instituto Agronômico, 1996. p. 237-239 (Boletim Técnico 100).

TASSO JÚNIOR, L. C.; SILVA NETO, H. F.; SILVA, J. D. R.; MARQUES, D.;

CAMILOTTI, F. Variação na fertilidade de solo ao longo da safra 2008/2009, para cultivares precoces de cana-de-açúcar. Enciclopédia Biosfera, Goiânia, v. 6, n.1, p. 1-9, 2010. 
